\section{Synthesis of Cost Effective and Sustainable Pathways to Wastewater Treatment}

\section{E. A. Aboagye ${ }^{1}$, C. $\operatorname{Tran}^{1}$, M. Desai ${ }^{1}$, K. M. Yenkie ${ }^{1}$, and F. Friedler ${ }^{2}$}

${ }^{1}$ Department of Chemical Engineering, Henry M. Rowan College of Engineering, Rowan University, Glassboro, NJ, 08028, USA

2Institute for Process Systems Engineering and Sustainability, Pazmany Peter Catholic University, Budapest, Hungary

Sustainable Design and Systems Medicine Lab

Group Website: https://yenkiekm.com/

Email: yenkie@rowan.edu 


\section{Global Water Resources \& Consumption}

- Very small percentage of the total water available is freshwater

- Yet, we depend on freshwater for our municipal, agricultural and industrial processes

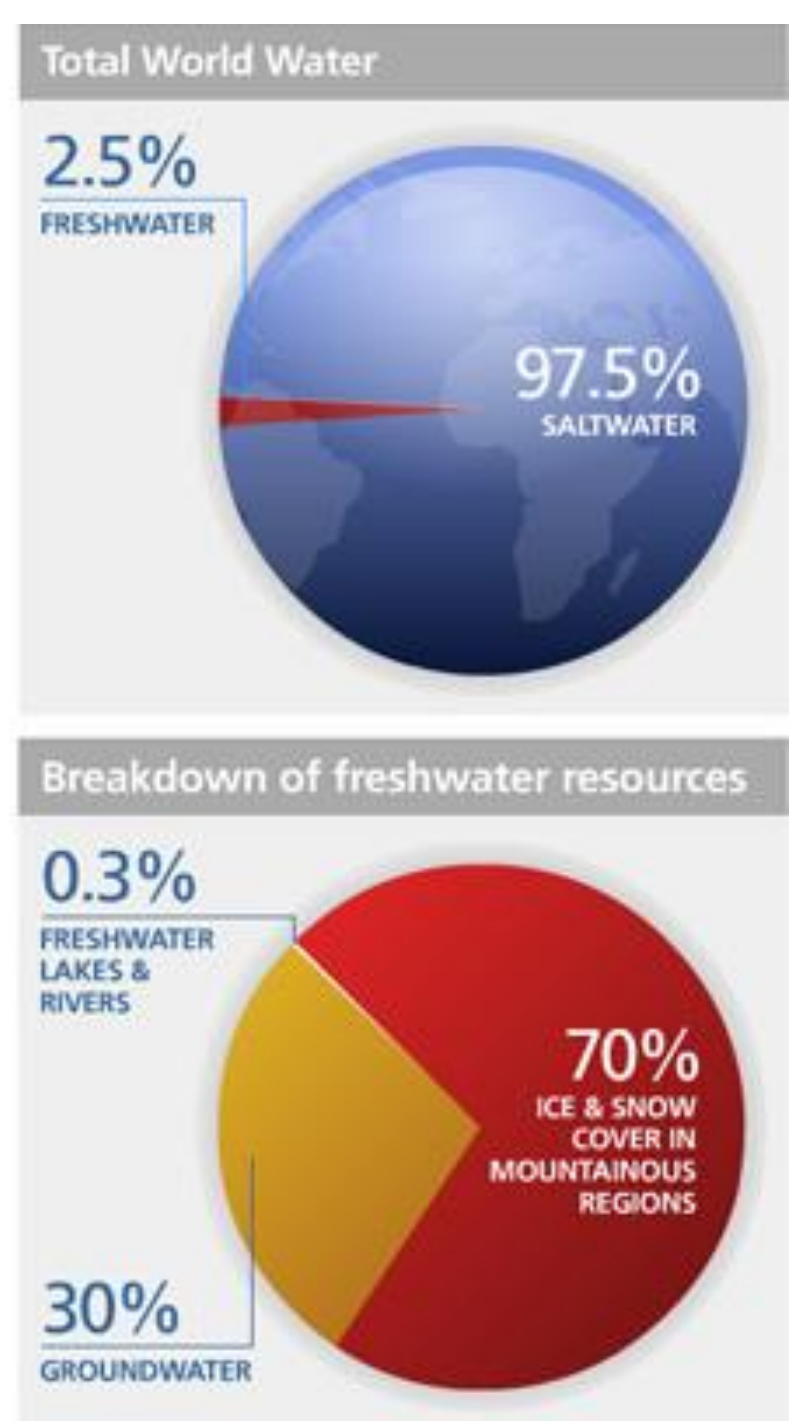

Global Water Consumption 1900 - 2025

(by region, in billions of $\mathrm{m}^{3}$ per year)

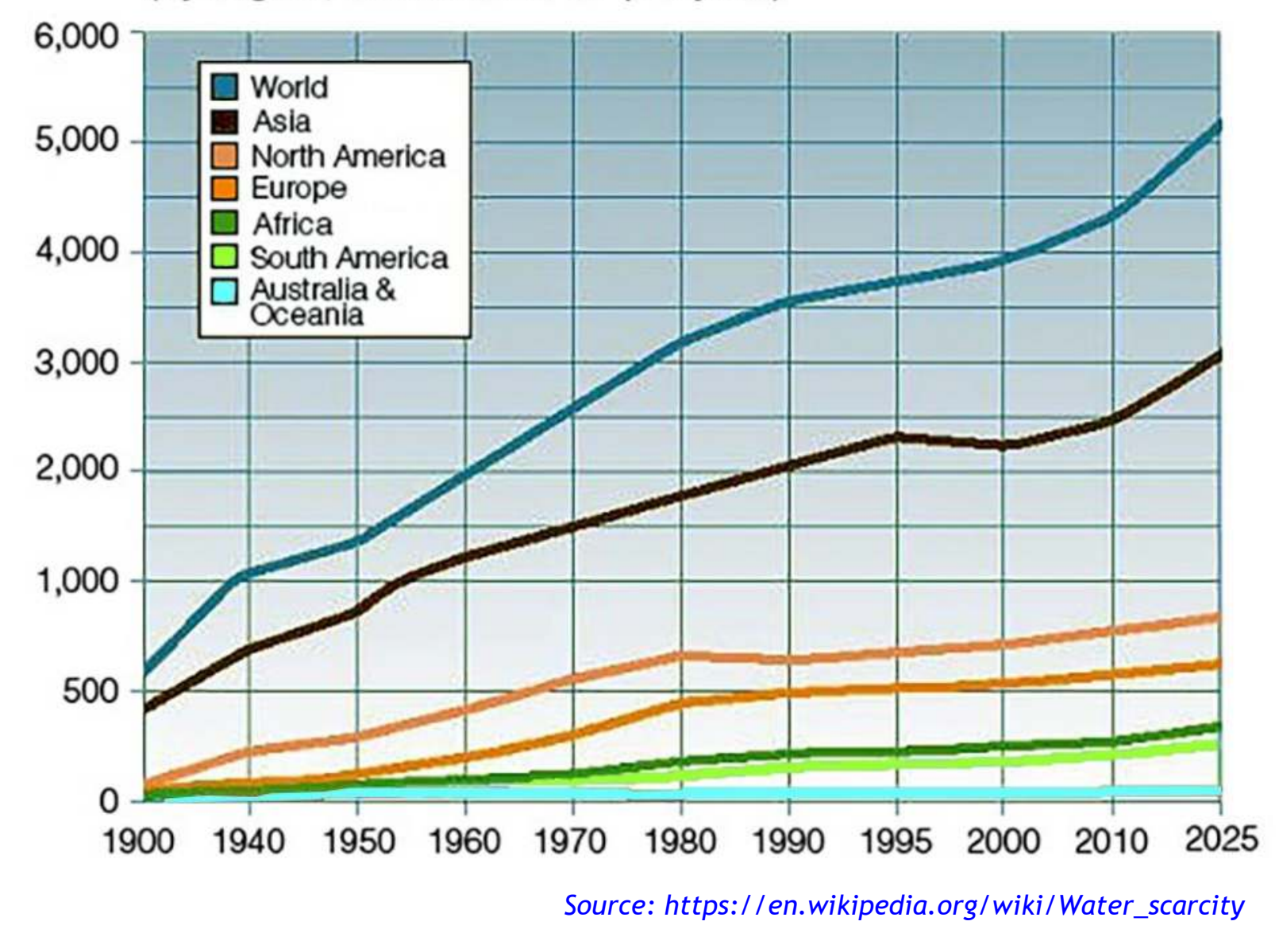




\section{Freshwater Usage Data}

$\underset{\text { University }}{\text { Rowan }}$

Current freshwater usage statistics (2018)

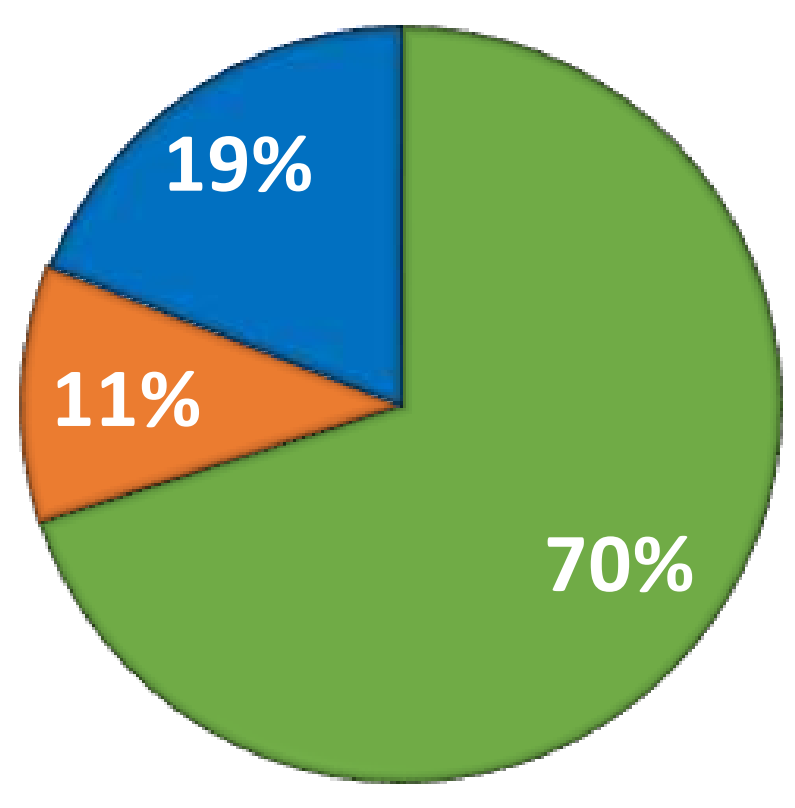

$\square$ Agricultural $\quad$ Municipal $\quad$ Industrial
Projected freshwater usage in $\mathbf{2 0 3 0}$

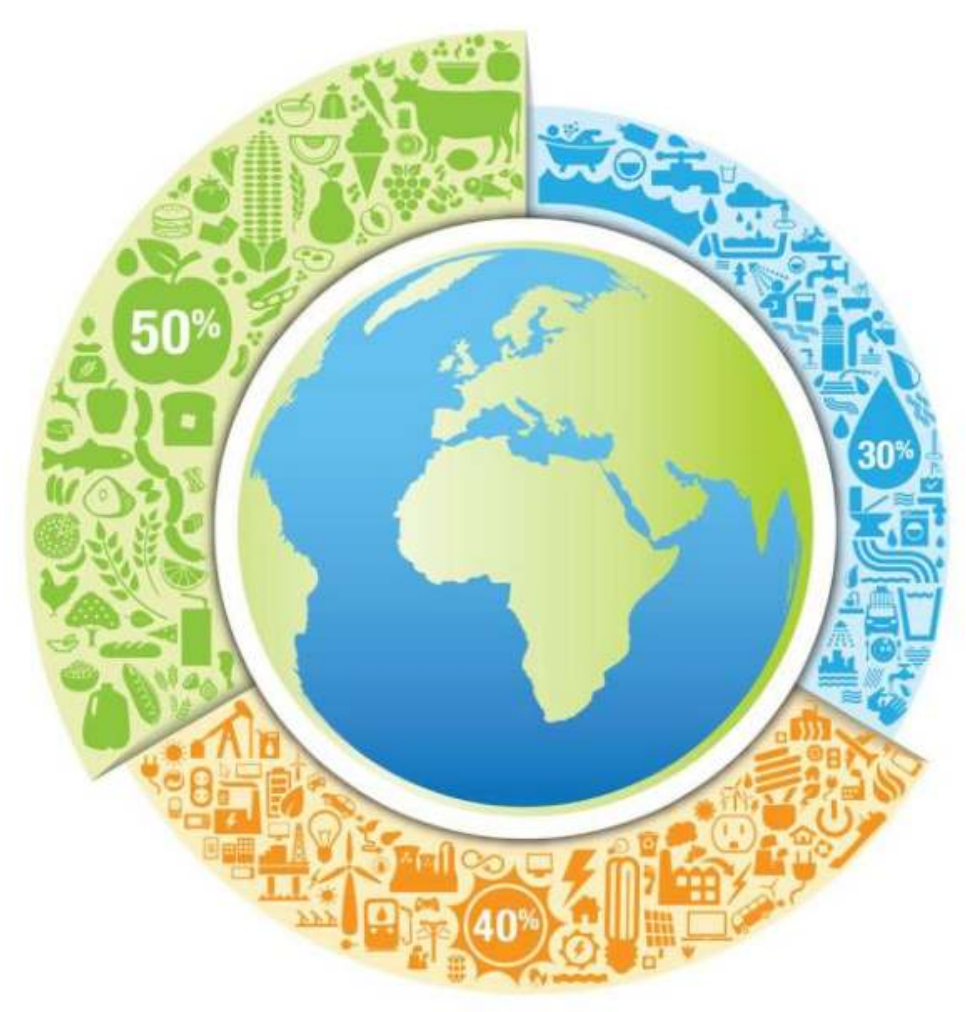

Where will this additional 20\% come from? 


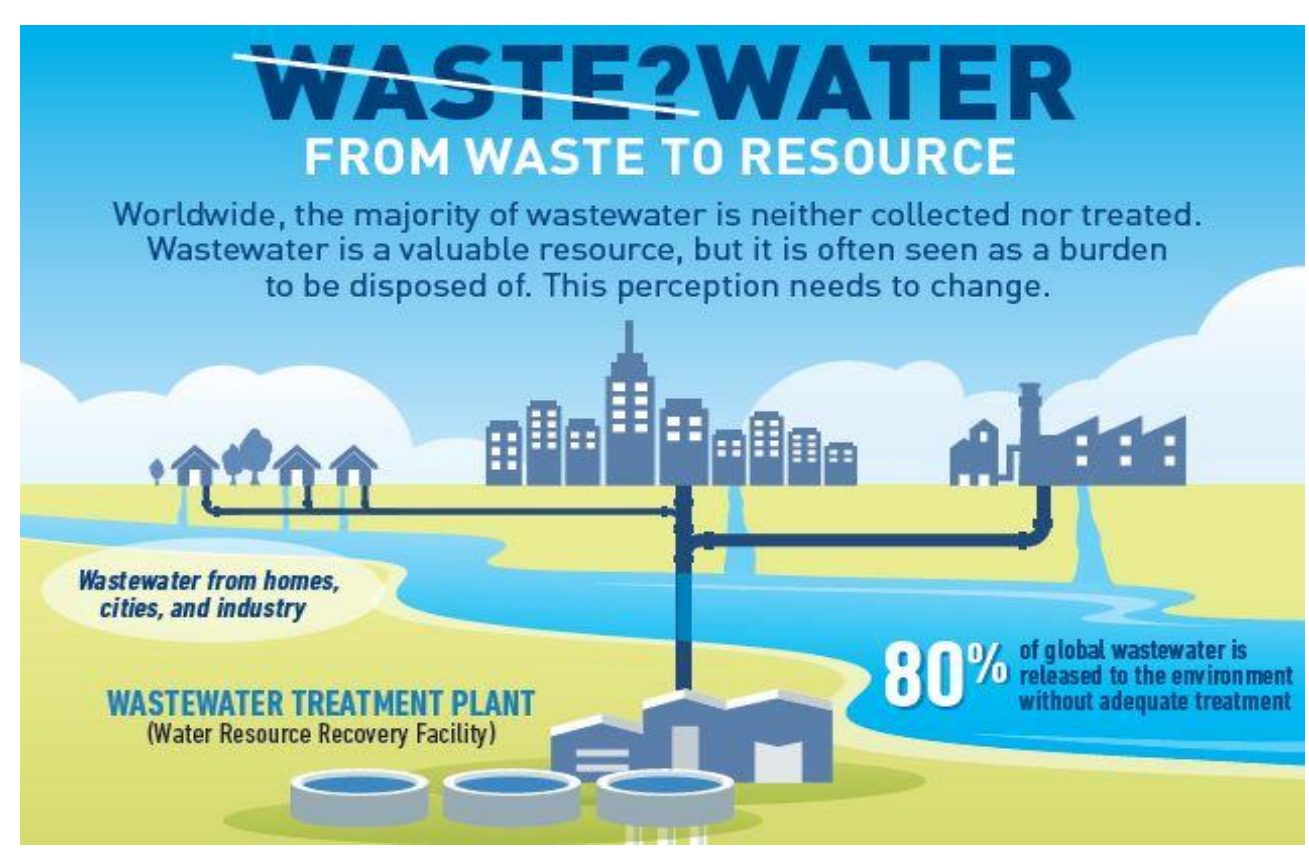
demands for sustainable existence tomorrow

- Systems Engineering can help in providing sustainable and cost effective insights

- To meet the demand we have to rely on treated and recycled water

- Thus, we need efficient wastewater treatment methods today to satisfy

Data Analysis

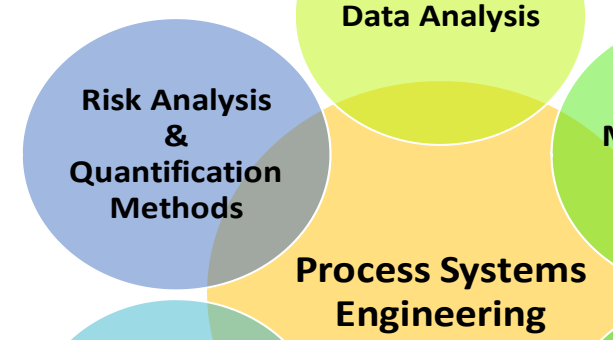

Optimization Process Systems Modeling

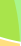

Process Design \& Operation 


\section{Wastewater Treatment: A Systems Problem}
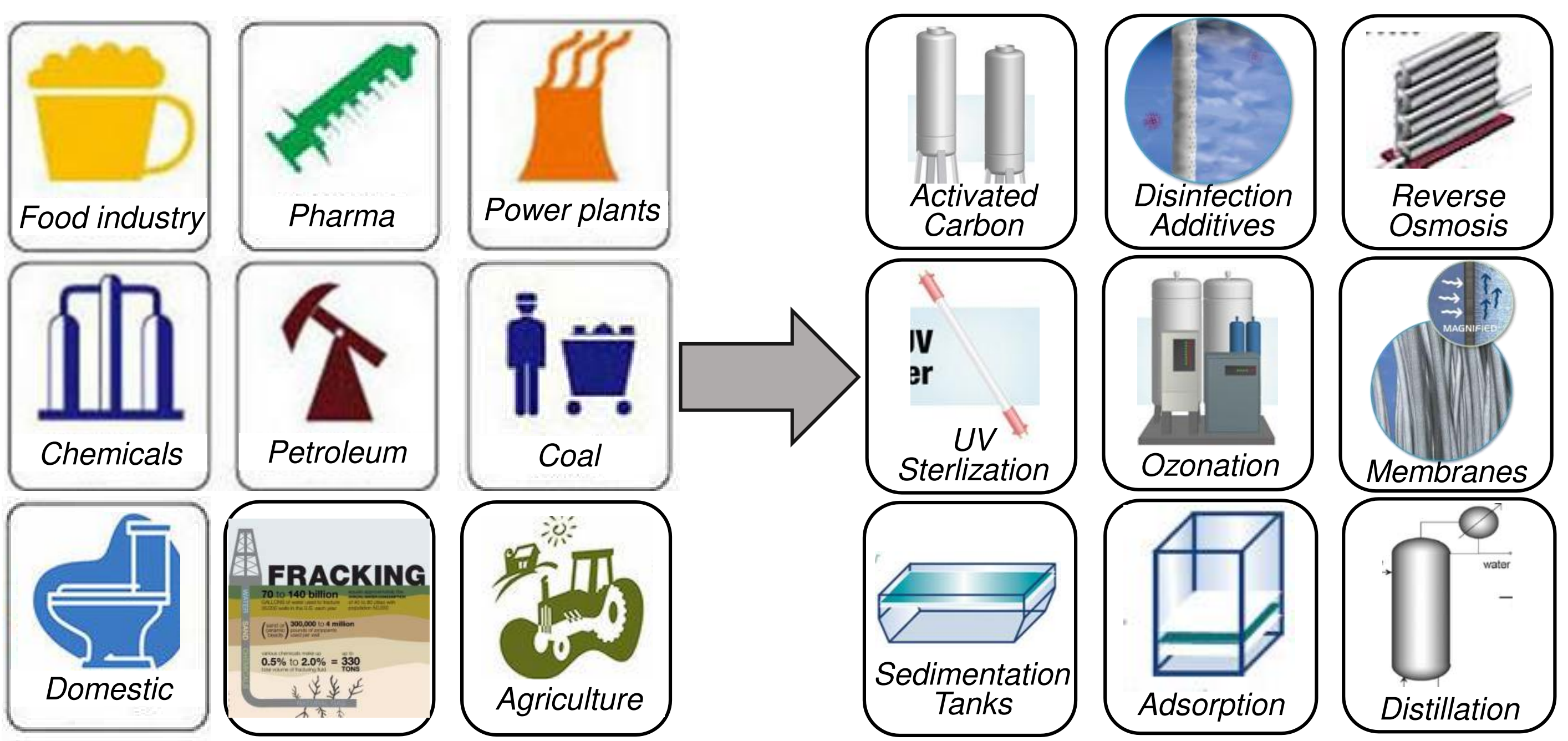

Sources of Wastewater

Treatment Methods 


\section{Designing a Treatment Process}

Solids removal is one of the tasks in Wastewater Treatment

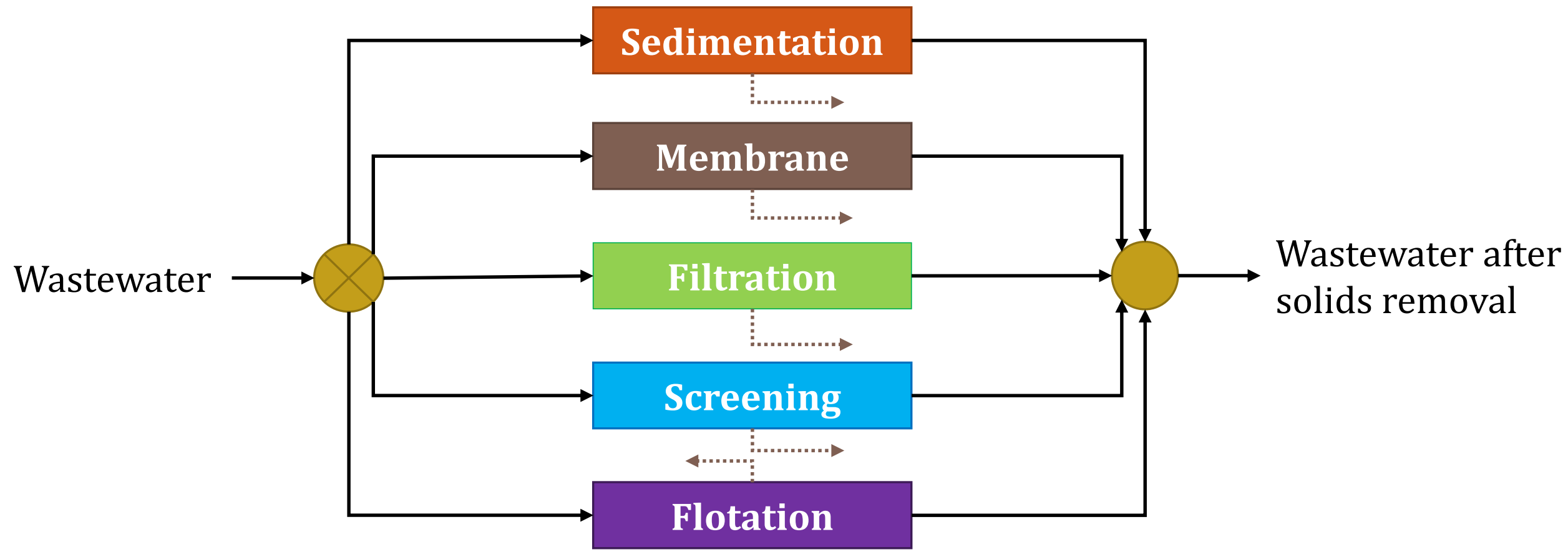

\section{Complexity of the design problem}

If a wastewater stream has $\mathbf{C}$ contaminants and $\mathbf{S}$ technologies for each task, then number of distinct treatment networks $\left(N_{f}\right)$ possible is given by

$$
N_{f}=\frac{[2(C-1)] ! S^{(C-1)}}{C !(C-1) !}
$$

If $C=5$ and $S=10$, then $N_{f}=1.4 \times 10^{5}$ (close to a million) possible networks 
- Wastewater treatment is most effective using a stagewise approach

- Technologies are placed is stages based on their contaminant removal capability

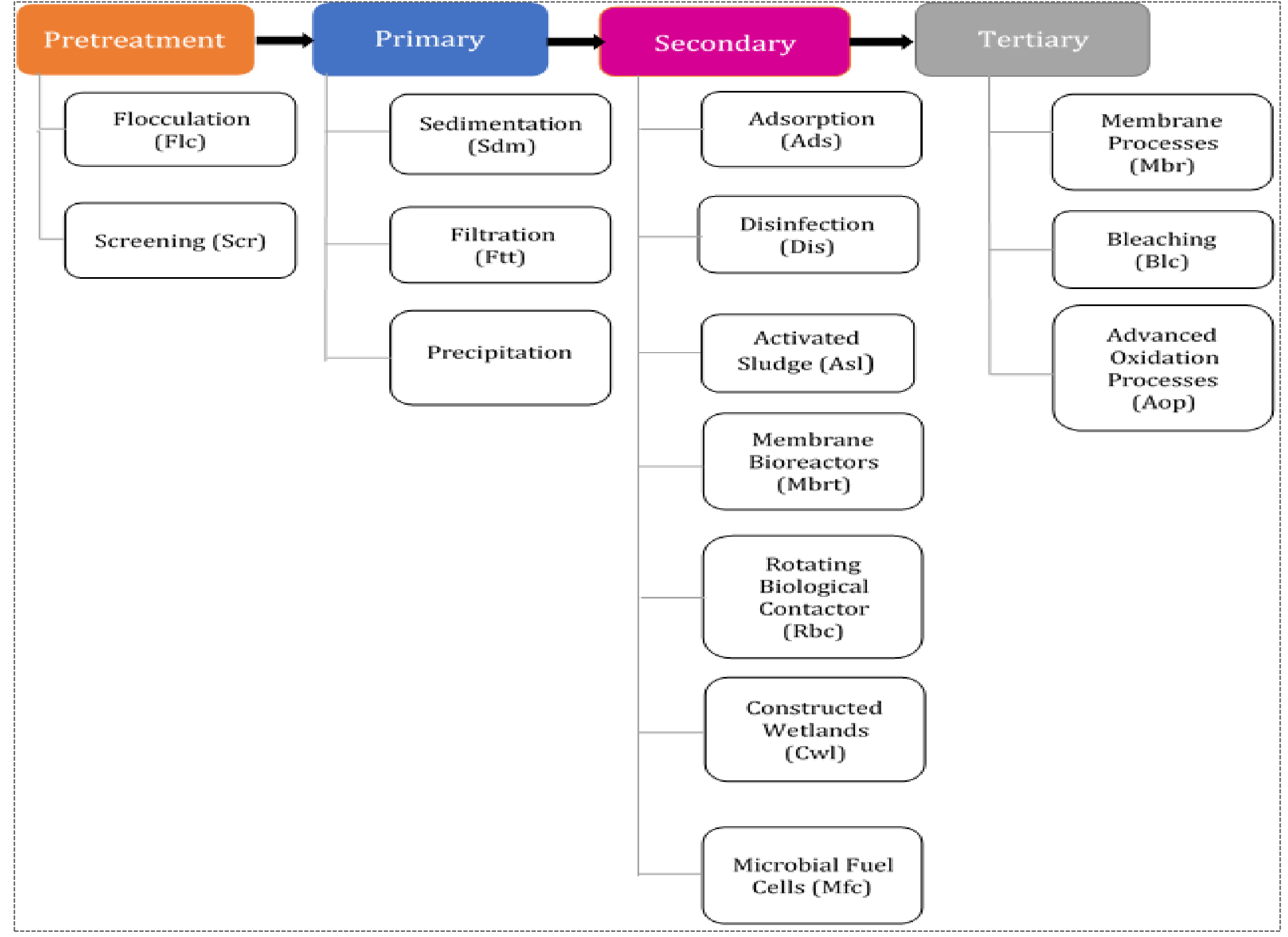




\begin{tabular}{|c|c|c|}
\hline Technology & Driving Force & Process conditions \\
\hline $\begin{array}{l}\text { Sedimentation }(\mathrm{Sdm}) \\
\text { or Decantation }(\mathrm{Dct})\end{array}$ & $\begin{array}{l}\text { Density gradient } \\
\text { Particle settling velocity }\end{array}$ & $\begin{array}{l}\text { Size, density of particle } \\
\text { Tank depth } \\
\text { Residence time }\end{array}$ \\
\hline Filtration (Ftt) & Particle size & $\begin{array}{l}\text { Average flux, } \\
\text { Pressure gradient } \\
\text { Filtration rate }\end{array}$ \\
\hline $\begin{array}{l}\text { Advanced Oxidation } \\
\text { Process (Aop) }\end{array}$ & $\begin{array}{l}\text { Oxidation reactions for contaminant } \\
\text { degradation }\end{array}$ & $\begin{array}{l}\text { Ozone, peroxide, } \\
\text { UV reactor }\end{array}$ \\
\hline Disinfection (Dis) & Chemical, radiation & $\begin{array}{l}\text { Chlorine dosage, Acid/Alkali treatment, } \\
\text { UV radiation }\end{array}$ \\
\hline $\begin{array}{l}\text { Rotating Biological } \\
\text { Contactors (Rbc) }\end{array}$ & Biological mechanism & $\begin{array}{l}\text { Biological film on rotational discs, speed of } \\
\text { rotation, aeration rate }\end{array}$ \\
\hline Membranes (Mbr) & $\begin{array}{l}\text { Particle/molecular size } \\
\text { Sorption/Diffusion } \\
\text { Pressure }\end{array}$ & $\begin{array}{l}\text { Pore size, Mol. wt. cut-off } \\
\text { Average flux, Pressure gradient } \\
\text { Type of membranes - MF, UF, NF and RO }\end{array}$ \\
\hline Activated Sludge (Acs) & Microbial activity & Detention time, mixing efficiency, aeration rate \\
\hline
\end{tabular}


- P-graph (process graph) studio uses vertices and nodes to represent materials \& operating units connected by arcs represent streams

- Includes two types of nodes

$\checkmark$ M-type: Materials

raw materials $\bigcirc$; products (O) \& intermediates

$\checkmark$ O-type: Operating units

- Based on axioms for structurally feasible process networks

- Algorithms available:

$\checkmark \quad$ MSG (Maximal Structure Generator): generates the maximal structure

$\checkmark \quad$ SSG (Solution-Structure Generator): generates all the structurally feasible process networks

$\checkmark \quad$ ABB (Accelerated Branch and Bound): generates the optimal or $n$-best feasible networks 


\section{Stage-wise Wastewater Treatment Superstructure}

Key:

Pretreatment; Primary

Treatment; Secondary Treatment; Tertiary

Treatment; Bypass

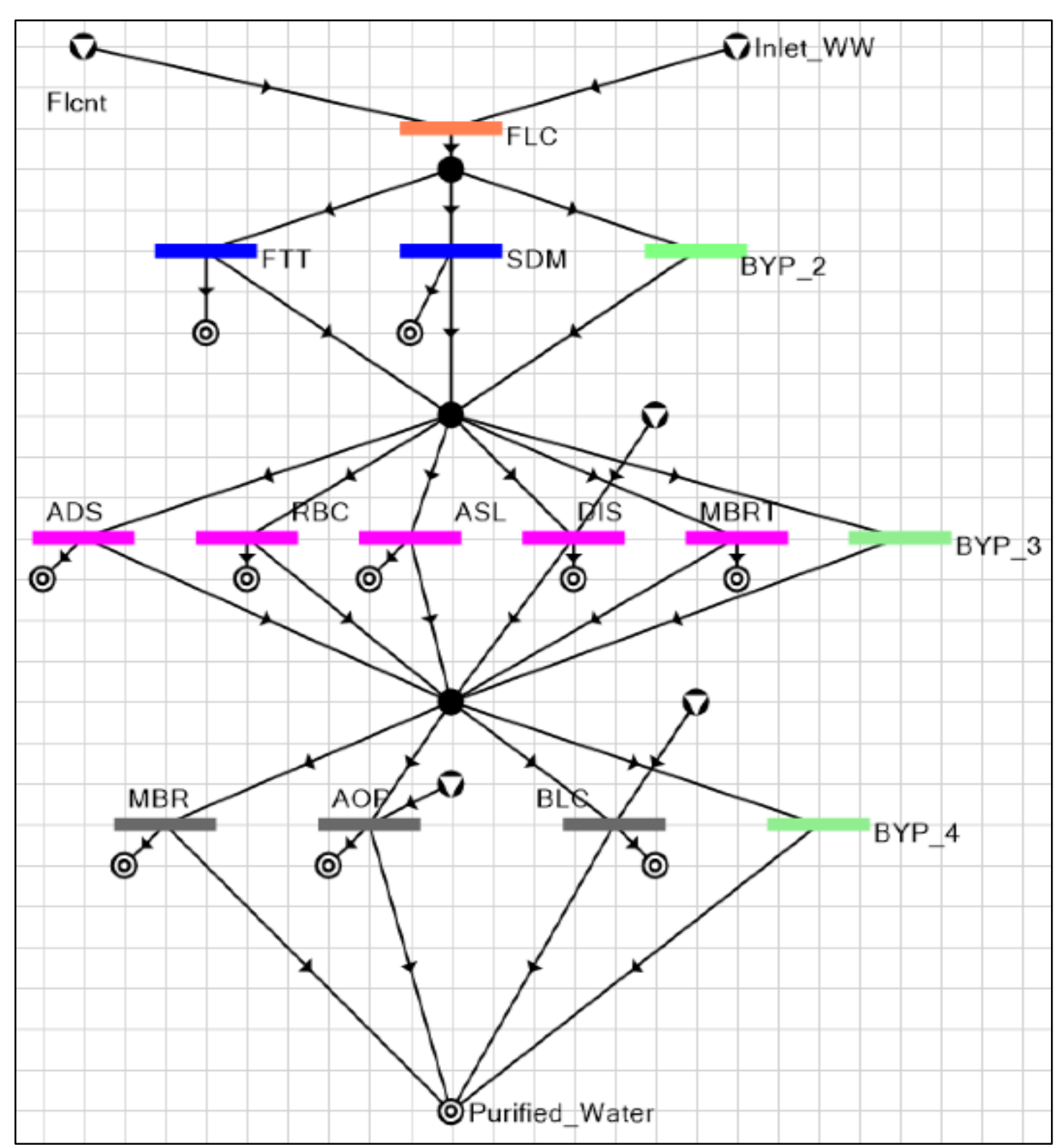

Superstructure Generated: P-Graph
What is a superstructure?

It is a systematic representation of all possible treatment technologies available in the 4 stages of wastewater treatment.

It also shows the flow from the initial wastewater stream towards the final purified water stream

FLC - Flocculation, SDM -

Sedimentation, FTT - Filtration, ADS Adsorption, RBC - Rotating Biological Containers, ASL - Activated Sludge, DIS - Disinfection, MBRT - Membrane Bioreactor, MBR - Membrane Processes, AOP - Advanced Oxidation Processes, BLC - Bleaching, BYP - Bypasses 


\section{Framework for Design \& Evaluation}

- Provide input wastewater composition and define output purity specifications

- Construct the wastewater treatment network design as an optimization model

$\checkmark$ Mass and energy balances

$\checkmark$ Investment and Operating Costs

$\checkmark$ Integer variables to select technologies by holding a $(0,1)$ value

- Method: P-Graph Studio (Graph Theory based Process Synthesis);

MILP (Mixed integer linear programming) solver 


\section{Case Study: Municipal Wastewater Treatment}

Entering flowrate of wastewater in the treatment system: $100 \mathrm{~m}^{3} / \mathrm{hr}$ Operating days per year: 330

Wastewater contaminant concentrations and purity levels

\begin{tabular}{|c|c|c|c|}
\hline Contaminant & Inlet Amount & Outlet Amount & Units \\
\hline Acids/Chlorides & 5000 & 300 & $\mathrm{mg} / \mathrm{L}$ \\
\hline COD (Inorganic) & 2000 & 70 & $\mathrm{mg} / \mathrm{L}$ \\
\hline BOD (Organic) & 1100 & 25 & $\mathrm{mg} / \mathrm{L}$ \\
\hline Settable solids & 200000 & 500 & $\mathrm{mg} / \mathrm{L}$ \\
\hline Metals (Pb, Cu, Ni, Zn) & 1340 & 5 & $\mu \mathrm{g} / \mathrm{L}$ \\
\hline
\end{tabular}




\section{Solutions from P-Graph formulation}

* The total best network solution generated using the P-Graph approach is 45 with a solution time of 0.035 seconds

* Costs: Fix and Proportional Investment Costs, Fix and Proportional Operating Costs

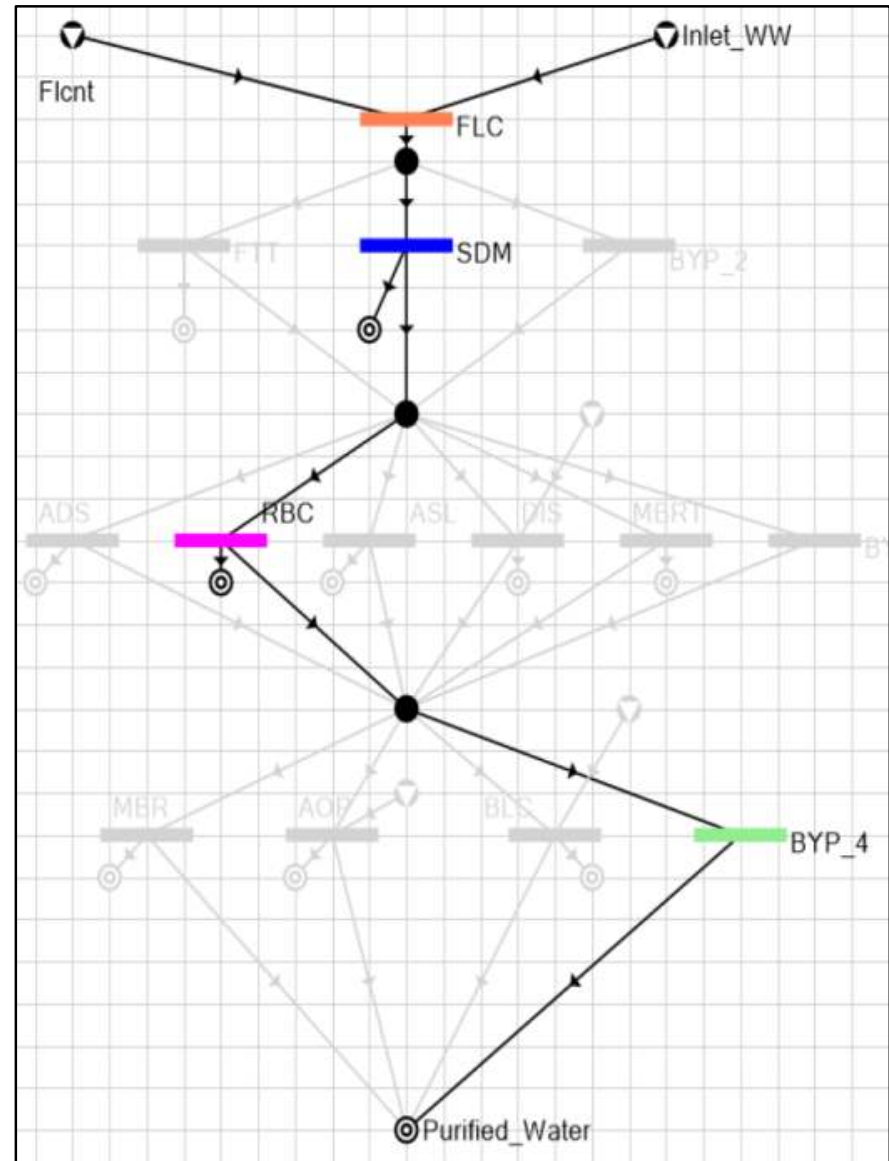

- Feasible Structure 1: (FLC-SDM-RBC)

- Purification Cost $\left(\$ / y r_{.}\right)$: 3197040

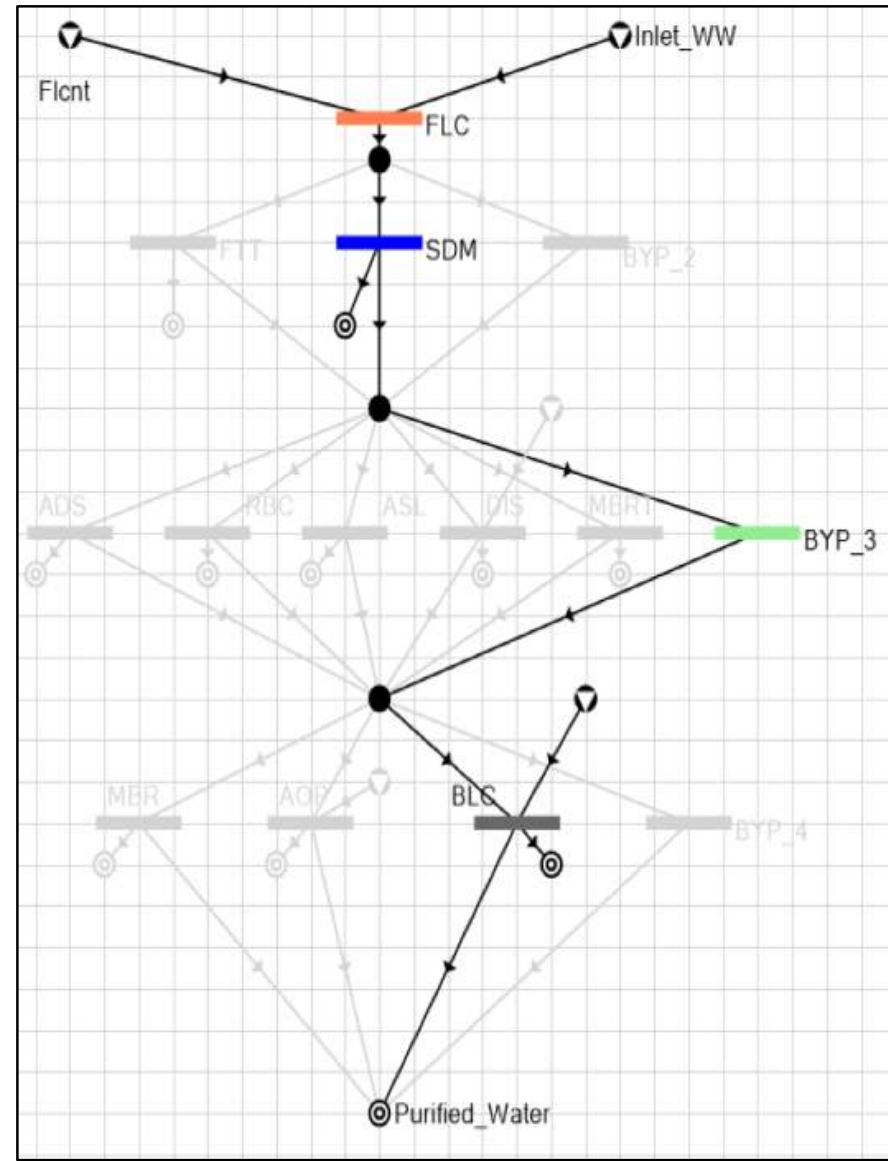

- Feasible Structure 2: (FLC-SDM-BLC)

- Purification Cost (\$/yr.) : 3256250

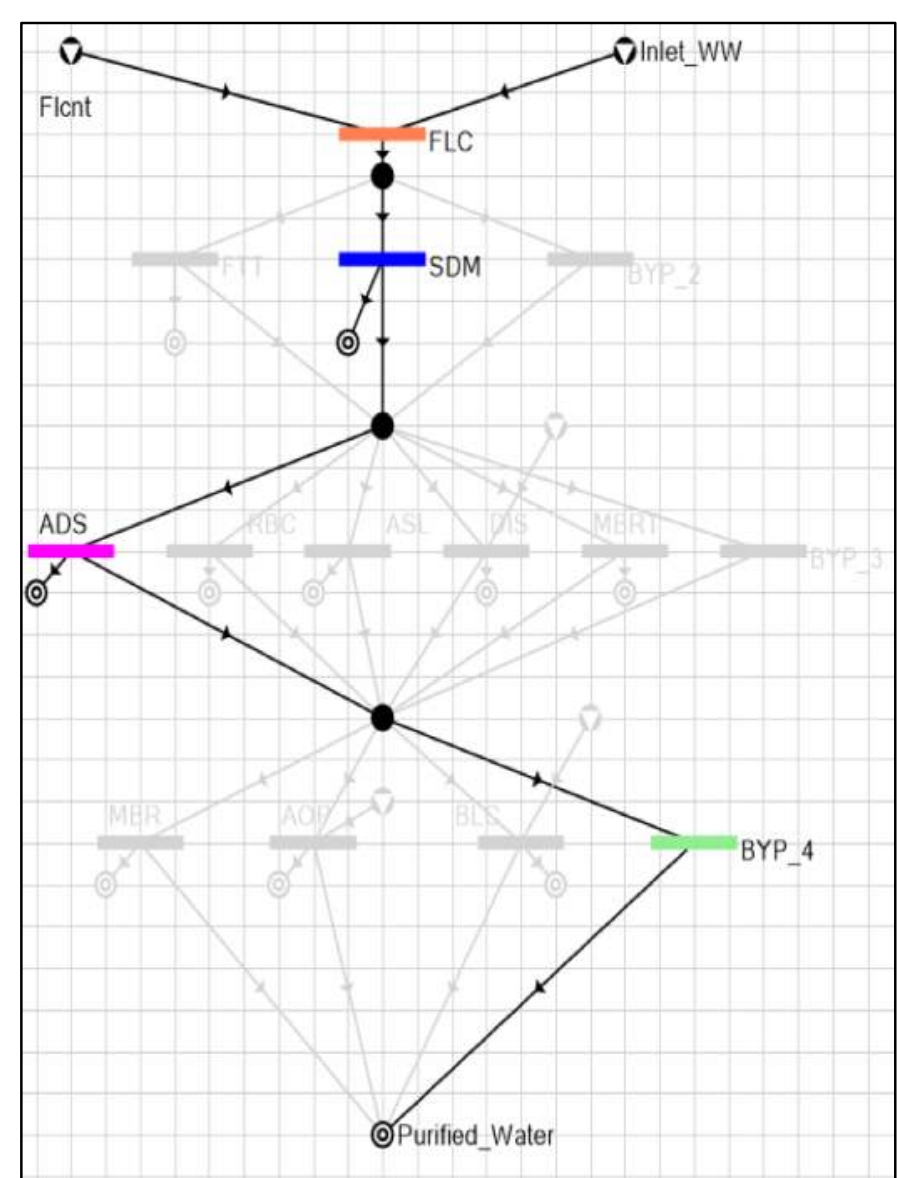

- Feasible Structure 3 (FLC-SDM-ADS)

- Purification Cost (\$/yr.) : 3901470 


\section{Solutions from P-Graph formulation}

Summary of the first best 10 feasible network pathways

\begin{tabular}{c|c|c|c}
\hline No & Network Path & $\begin{array}{c}\text { Purification } \\
\text { Cost, }(\$ / y r)\end{array}$ & $\begin{array}{c}\text { Purification Cost/Liter of Purified } \\
\text { Water(\$/L) }\end{array}$ \\
\hline 1 & FLC-SDM-RBC & 3197040 & 0.004249 \\
2 & FLC-SDM-BLC & 3256250 & 0.004327 \\
3 & FLC-SDM-ADS & 3901470 & 0.005185 \\
4 & FLC-FTT & 4447680 & 0.005637 \\
5 & FLC-SDM-RBC-BLC & 4507400 & 0.005990 \\
6 & FLC-SDM-AOP & 4815100 & 0.006399 \\
7 & FLC-SDM-ADS-BLC & 5343310 & 0.007101 \\
8 & FLC-FTT-RBC & 5417120 & 0.007199 \\
9 & FLC-FTT-BLC & 5482110 & 0.007286 \\
10 & FLC-SDM-RBC-AOP & 6066250 & 0.008062 \\
\hline
\end{tabular}




\section{The Sustainable Process Index (SPI)}

- Ecological Footprint

- Quantification of sustainability based on arable area needed to produce one unit of product

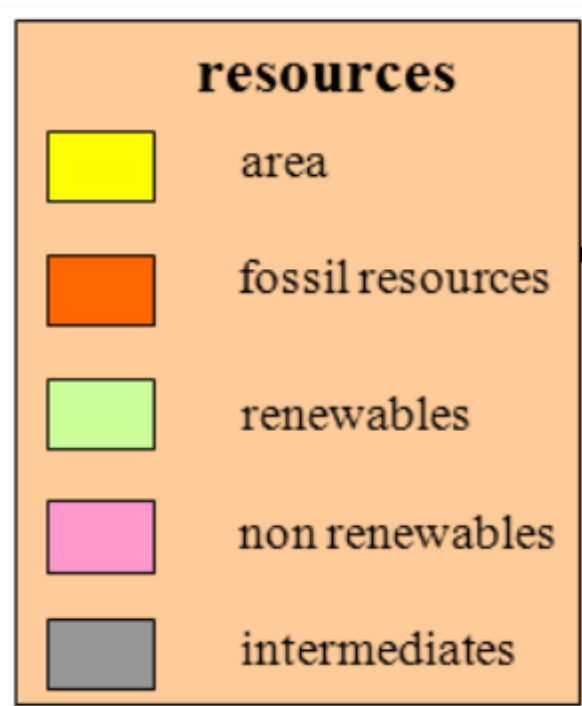

- The burden of a process on the ecosystem

- The higher the SPI, the less sustainable the process is
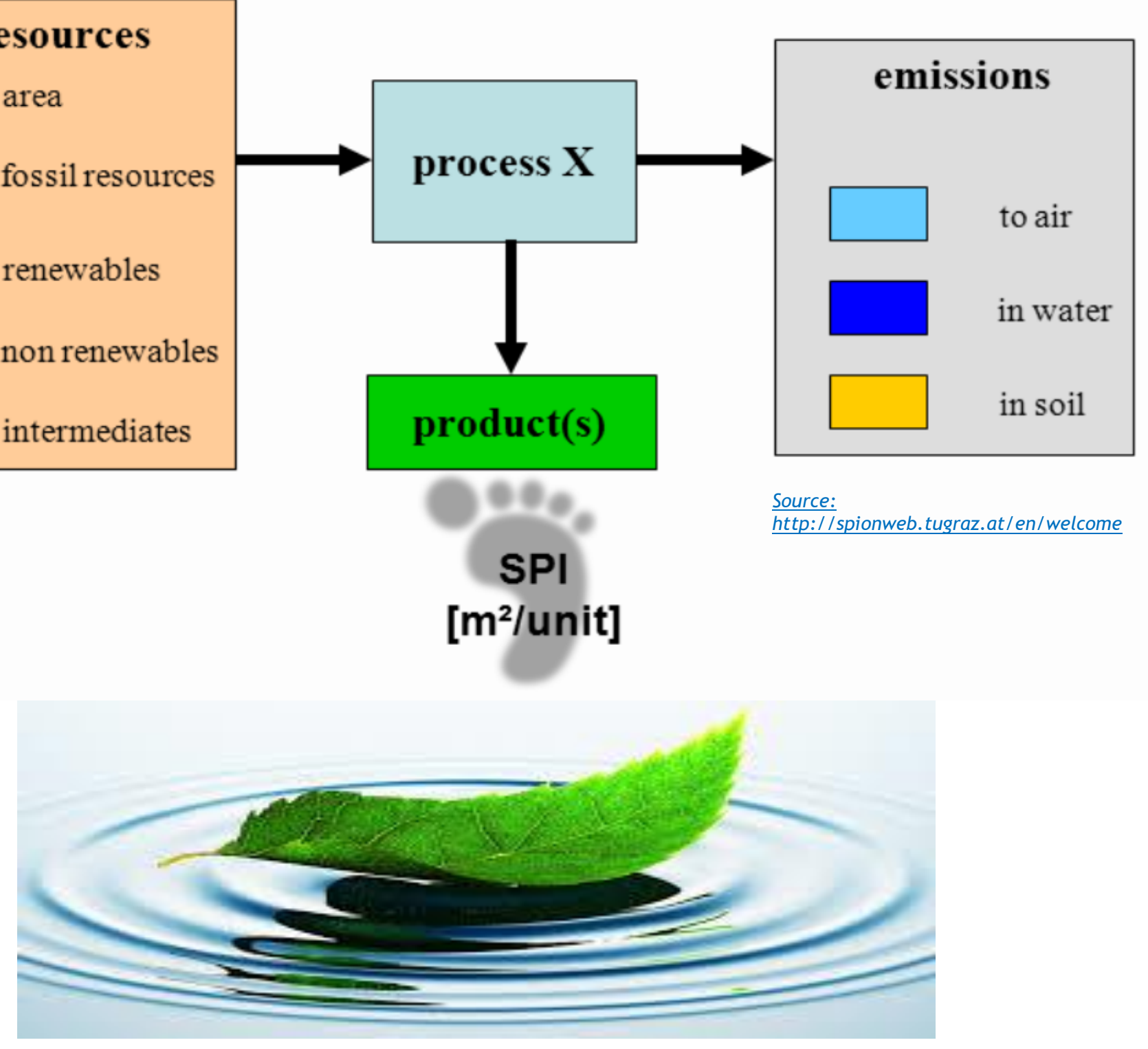


\section{Solutions from SPI}

Rowan
University

- FLC-SDM-RBC-AOP (7) has the highest SPI Value

- FLC-FTT (4) has the lowest SPI Value

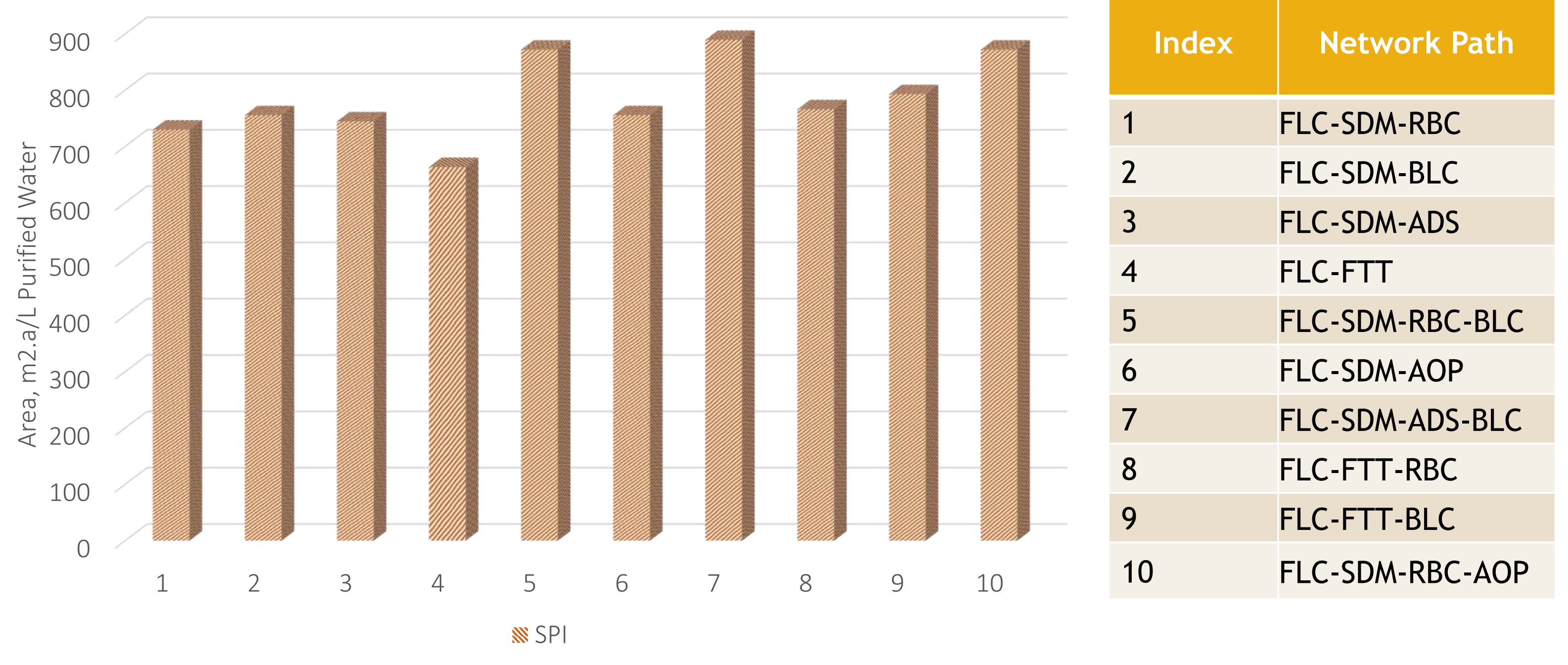




\section{Conclusions from P-Graph and SPI Analysis}

- In terms of cost, network path (1) had the minimum, but network path (4) had had the lowest SPI value

- Even though network path (4) had the lowest SPI value, it was the only network path that could not meet purification requirements

- To make a decision, there should be trade-offs between cost and "greenness" of the process 
* An integrated approach involving design and optimization for generation of cost-effective wastewater treatment networks

* P-Graph approach to wastewater treatment using a superstructure optimization (MINLP)

* Generated a rank of network synthesis for wastewater treatment based on purification cost

* Sustainable process index (SPI) evaluation of generated synthesis paths 


\section{ACS}

Chemistry for Life ${ }^{\circledR}$

AMERICAN Chemical Society

\section{Thank you for your attention!}

